Pacific Journal of Mathematics

THE BRAVER GROUP OF POLYNOMIAL RINGS 


\title{
THE BRAUER GROUP OF POLYNOMIAL RINGS
}

\author{
F. R. DEMEYER
}

Let $R$ be a commutative ring and $S$ a commutative $R$ algebra. The induced homomorphism $B(R) \rightarrow B(S)$ of Brauer groups is studied for the following choices of $S$. First, $S=$ $R / I$ where $I$ is an ideal in the radical of $R$. Second, $S=$ $R[x]$ the ring of polynomials in one variable over $R$. Third, $S=K$ the quotient field of $R$ when $R$ is a domain.

In [3] M. Auslander and O. Goldman introduced the Brauer group $B(R)$ of a commutative ring $R$. If $S$ is a commutative $R$-algebra there is a homomorphism $B(R) \rightarrow B(S)$ induced by the homomorphism from $R$ to $S$. Some of the choices for $S$ considered in [3] are $S=$ $R / I$ for an ideal $I$ of $R$, or $S=K$ the quotient field of $R$ when $R$ is a domain, or $S=R[x]$ the ring of polynomials in one variable over $R$.

We observe here relationships between the homomorphisms of Brauer groups induced from these choices for $S$. We show that if $I$ is an ideal in the radical of $R$ and $R$ is complete in its $I$-adic topology then $B(R) \cong B(R / I)$. This answers a question raised in [11]. If $I$ is a nil ideal in $R$ then $B(R) \cong B(R / I)$. If $R[[x]]$ is the ring of formal power series over $R$ then $B(R[[x]]) \cong B(R)$. If we assume $R$ is a domain with quotient field $K$ an algebraic number field and $t_{1}, \cdots, t_{n}$ are indeterminates the homomorphism $B\left(R\left[t_{1}, \cdots, t_{n}\right]\right) \rightarrow$ $B\left(K\left(t_{1}, \cdots, t_{n}\right)\right)$ is a monomorphism where $K\left(t_{1}, \cdots, t_{n}\right)$ is the function field in $n$-variables over $K$. Let $B^{\prime}(R[x])$ be the kernel of the natural homomorphism $B(R[x]) \rightarrow B(R)$ where $x$ is an indeterminate. If $R$ is a domain there is a procedure given in [13] for calculating $B^{\prime}(R[x])$ in terms of $B^{\prime}(\bar{R}[x])$ where $\bar{R}$ is the integral closure of $R$. In [3] it is shown that $B^{\prime}(R[x])=0$ if $R$ is a regular domain of characteristic = 0 . We fill in the gap between these two results in the Noetherian case.

If $R$ is an integrally closed Noetherian domain, let Ref $(R)$ denote the isomorphism classes of finitely generated reflexive $R$-modules $M$ with $\operatorname{End}_{R}(M)$ projective over $R$ and let Pro $(R)$ be the projective elements in $\operatorname{Ref}(R)$. Under the multiplication $|M| \cdot|N|=\left|(M \otimes N)^{* *}\right|$ $\operatorname{Ref}(R)$ is a monoid, $\operatorname{Pro}(R)$ is a submonoid and $\operatorname{Ref}(R) / \operatorname{Pro}(R)$ is a group (see [6]). There is a split exact sequence.

$0 \rightarrow \operatorname{Ref}^{\prime}(R[x]) \rightarrow \operatorname{Ref}(R[x]) / \operatorname{Pro}(R[x]) \rightarrow \operatorname{Ref}(R) / \operatorname{Pro}(R) \rightarrow 0$ where $\operatorname{Ref}^{\prime}(R[x])=\operatorname{Ref}(R[x]) /(\operatorname{Pro}(R[x])+\operatorname{Ref}(R))$. Utilizing results in [1] we show that the sequence.

$0 \rightarrow \operatorname{Ref}^{\prime}(R[x]) \rightarrow B^{\prime}(R[x]) \rightarrow B^{\prime}(K[x])$ is exact. If $R$ is any von 
Neumann regular ring then $B^{\prime}(R[x])$ is trivial if and only if $R / m$ is a perfect field for each maximal ideal $m$ of $R$. If $R$ is a Boolean ring, $B(R[x])=(0)$.

We adapt an example from [14] to obtain a Noetherian domain $R$ with quotient field $K$ such that $B(R) \rightarrow B(K)$ is monomorphism but $B(R[x]) \rightarrow B(K(x))$ has an infinite kernel. We also give some examples of domains $R$ containing a prime ideal $I$ such that the homomorphism $B(R) \rightarrow B(R / I)$ is not onto.

Throughout $R$ denotes a commutative ring and unless otherwise specified $\otimes$ means $\otimes_{R}$. An Azumaya algebra is called central separable in [3] and [10].

Otherwise all our undefined terminology, conventions, and notations are as in [10]. This paper was written while the author visited at the Forschungsinstitut für Mathematik in Zürich. The author thanks the people of the Institute, and especially Professor M. Knus for a sympathetic ear.

1.

LEMMA 1. Let $I$ be an ideal in the radical of a commutative ring $R$. If for any Azumaya $R$-algebra $A$ idempotents can be lifted from $A / I A$ to $A$ then the induced homomorphism $B(R) \rightarrow B(R / I)$ is a monomorphism.

In [9] we showed that if $I$ is nilpotent then $B(R)$ is isomorphic to $B(R / I)$. The proof there that $B(R) \rightarrow B(R / I)$ is a monomorphism also proves Lemma 1 . See also [16].

THEOREM 2. Let $I$ be an ideal in the radical of a commutative ring $R$. If $R$ is complete in its I-adic topology then the homomorphism $B(R) \rightarrow B(R / I)$ is an isomorphism.

Proof. Let $A$ be a Azumaya $R$-algebra. Since $R$ is $I$-adically complete and $A$ is finitely generated as an $R$-module, we have $A$ is complete with respect to the ideal $I A$. Thus idempotents can be lifted from $A / I A$ to $A$ so by Lemma $1 B(R) \rightarrow B(R / I)$ is a monomorphism.

Next let $A_{0}$ be a Azumaya $R / I$-algebra. We showed in [9] in proving that $B(R) \rightarrow B(R / I)$ is onto when $I$ is nilpotent that one can construct a sequence $A_{i}$ of Azumaya algebras over $R / I^{2^{i}}$ with $A_{i} / I^{2^{i-1}} A_{i} \cong$ $A_{i-1}$ by a natural homomorphism $\varphi_{i}$. We let $A=\lim \left(A_{i}\right)$. Then $A$ is an $R$-algebra with $A / I^{2^{i}} \cong A_{i}$. If $a \in A$ we let the natural image of $a$ in $A_{i}$ be $a^{i}$. Let $a_{1}, \cdots, a_{n}$ be elements of $A$ with $a_{1}^{0}, \cdots, a_{n}^{0}$ generating $A / I=A_{0}$ as an $R$-module. By Nakayama's lemma (Prop. 
2.2 p. 85 [5]) the elements $a_{1}^{i}, \cdots, a_{n}^{i}$ generate $A_{i}$ as an $R$-module for each $i$. Let $a \in A$. Then

$$
a^{0}=\sum_{j=1}^{n} \alpha_{j, 0} a_{j}^{0} \quad \alpha_{j, 0} \in R
$$

Thus

$$
a^{1}=\sum_{j=1}^{n} \alpha_{j, 0} a_{j}^{1}+x^{1} \quad \text { where } \quad x^{1} \in I\left(A / I^{2}\right),
$$

we can write

$$
x^{1}=\sum_{j=1}^{n} \gamma_{j, 0} a_{j}^{1} \quad \text { where } \quad \gamma_{j, 0} \in I
$$

Thus

$$
a^{1}=\sum_{j=1}^{n}\left(\alpha_{j, 0}+\gamma_{j, 0}\right) \alpha_{j}^{1} .
$$

Let $\alpha_{j, 1}=\alpha_{j, 0}+\gamma_{j, 0}$, then $a^{1}=\sum_{1=1}^{n} \alpha_{j, 1} a_{j}^{1}$ and $\alpha_{j, 1}-\alpha_{j, 0} \in I$. Continuing inductively we find

$$
a^{i}=\sum_{j=1}^{n} \alpha_{j, i} a_{j}^{i} \quad \text { with } \quad \alpha_{j, i} \in R, \alpha_{j, i}-\alpha_{j, i-1} \in I^{2^{i-1}} .
$$

Let $\alpha_{j}=\lim _{i \rightarrow \infty} \alpha_{j, i}$ and let $b=\sum_{j=1}^{n} \alpha_{j} a_{j}$. For any $i, a^{i}=b^{i}$ so $b=a$ and $A$ is finitely generated as an $R$-module.

For any maximal ideal $m$ of $R, R / m \otimes A=R / m \otimes R / I \otimes A=$ $R / m \otimes A_{0}$. Thus $R / m \otimes A$ is separable over $R / m$. By Theorem 2.7.1. of [10], $A$ is separable over $R$. Let $Z(A)$ be the center of $A$, then $Z(A)$ is a direct summand of $A$ so $Z(A)$ is finitely generated over $R$. Moreover, $0 \rightarrow R / m \otimes Z(A) \rightarrow R / m \otimes A$ is exact so for every maximal ideal $m$ of $R, R / m \otimes Z(A) / R=0$. Thus $Z(A)=R$ and $A$ is an Azumaya $R$-algebra completing the proof.

CoRollary 3. Let $N$ be a nil ideal in the commutative ring $R$, then $B(R) \cong B(R / N)$.

Proof. The hypothesis of Lemma 1 are satisfied since idempotents can be lifted modulo a nil ideal so the map from $B(R)$ to $B(R / N)$ is one-to-one.

Let $\bar{A}$ be an Azumaya algebra over $R N=\bar{R}$. There is a finitely generated subring $\bar{S}$ of $\bar{R}$ and an Azumaya $\bar{S}$-algebra $\bar{B}$ with $\bar{B} \bigotimes_{\bar{s}} \bar{R}=\bar{A}$. Let $\bar{s}_{1}, \cdots, \bar{s}_{m}$ be the generators of $\bar{S}$ over its prime ring and let $s_{1}, \cdots, s_{m}$ be corresponding elements in $R$. Let $S$ be the subring of $R$ generated by $s_{1}, \cdots, s_{m}$. Now $N$ consists of nilpotent 
elements in $R$ so $N \cap S$ is a nil-ideal in $S$ and $S / N \cap S \cong \bar{S}$. Since $S$ is Noetherian, $N \cap S$ is nilpotent. As we saw in the proof of Theorem 2 there is an Azumaya $S$-algebra $B$ with $\bar{S} \bigotimes_{S} B=\bar{B}$. Let $A=R \otimes_{S} B$, then

$$
\bar{R} \otimes A \cong \bar{R} \otimes R \bigotimes_{S} B \cong \bar{R} \bigotimes_{S} B \cong \bar{R} \bigotimes_{\bar{S}} \bar{S} \bigotimes_{S} B \cong \bar{R} \bigotimes_{\bar{S}} \bar{B} \cong \bar{A}
$$

thus the homomorphism from $B(R)$ to $B(R / N)$ is onto.

Corollary 4. Let $R[[x]]$ be the ring of formal power series in $x$ over $R$, then $B(R) \cong B(R[[x]])$.

Proof. The ideal $(x)$ generated by $x$ is in the radical of $R[[x]]$ and $R[[x]]$ is complete in the topology induced by this ideal. Thus by Theorem $2, \mathrm{~B}(R) \cong B(R[[x]])$.

LEMma 5. Let $R$ be a commutative ring and $S$ a commutative $R$-algebra. Then the sequence

$$
0 \longrightarrow B(S[x] / R[x]) / B(S / R) \longrightarrow B^{\prime}(R[x]) \longrightarrow B^{\prime}(S[x])
$$

is exact and $B(S[x] / R[x]) \cong B(S / R) \oplus L$ for an abelian group $L$.

Proof. Consider the diagram of Brauer groups where the maps are all natural.

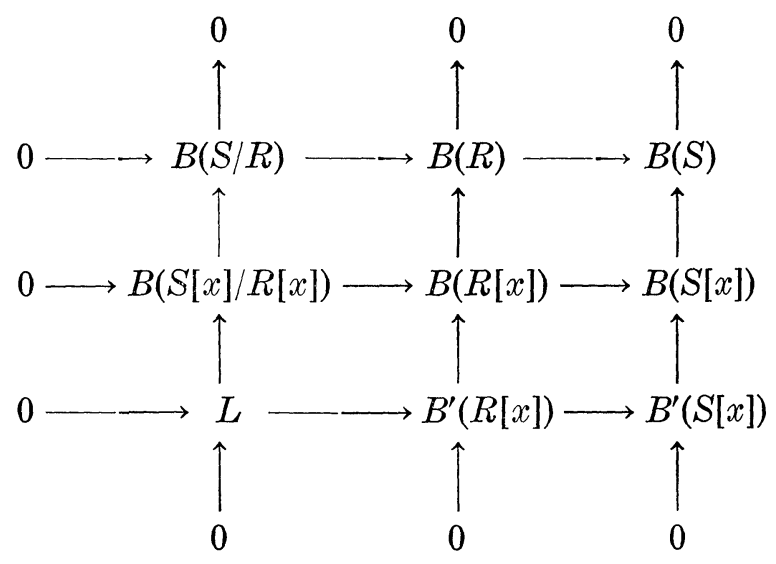

The first two horizontal rows are exact by definition and the last two vertical rows are split exact. The exactness of the third horizontal row follows as does the split exactness of the first vertical row. This gives the lemma.

Let $R$ be a domain with quotient field $K$. Let $K(x)$ be the field of fractions of $K[x]$. If $K$ is perfect then $L$ in Lemma 5 is $B^{\prime}(R[x])$ 
since $B^{\prime}(K[x])=0$ by Theorem 7.5 of [3]. Thus $B(K[x] / R[x]) \cong$ $B(K / R) \oplus B^{\prime}(R[x])$. Later we give an example where $B^{\prime}(R[x])$ is infinite and $B(K / R)=0$. If $K$ is any field then $0 \rightarrow B(K[x]) \rightarrow B(K(x))$ is exact so $B(K(x) / R[x]) \cong B(K[x] / R[x])$.

THEOREM 6. Let $R$ be a domain whose quotient field $K$ is an algebraic number field. Then $0 \rightarrow B\left(R\left[x_{1}, \cdots, x_{n}\right]\right) \rightarrow B\left(K\left(x_{1}, \cdots, x_{n}\right)\right)$ is exact.

Proof. The case $n=0$ is in [7]. By Corollary 3.9 of [14] $B^{\prime}\left(R\left[x_{1}\right.\right.$, $\left.\left.\cdots, x_{n-1}\right]\left[x_{n}\right]\right)=0$. Now $K\left(x_{1}, \cdots, x_{n}\right)$ is a perfect field thus $B\left(K\left(x_{1}\right.\right.$, $\left.\left.\cdots, x_{n}\right) / R\left[x_{1}, \cdots, x_{n}\right]\right)=B\left(K\left(x_{1}, \cdots, x_{n-1}\right) / R\left[x_{1}, \cdots, x_{n-1}\right]\right)$ the proof is complete by induction.

Next let $R$ be a Noetherian integrally closed domain. Let Ref $(R)$ denote the isomorphism classes of finitely generated reflexive $R$-modules $M$ with $\operatorname{End}_{R}(M)$ projective. Multiply two such classes by $|M| \cdot|N|=$ $\left|(M \otimes N)^{* *}\right|$ where $M^{*}=\operatorname{Hom}_{R}(M, R)$. Then $\operatorname{Ref}(R)$ is a commutative monoid. Let Pro $(R)$ be the submonoid of those $M$ which are projective. Then $\operatorname{Ref}(R) / \operatorname{Pro}(R)$ is a group (see [6]).

The natural homomorphism from $R[x]$ onto $R$ and its splitting map from $R$ to $R[x]$ induces a split exact sequence

$$
\begin{aligned}
& 0 \longrightarrow \operatorname{Ref}(R[x]) /(\operatorname{Ref}(R)+\operatorname{Pro}(R[x])) \longrightarrow \operatorname{Ref}(R[x]) / \operatorname{Pro}(R[x]) \\
& \longrightarrow \operatorname{Ref}(R) / \operatorname{Pro}(R) \rightarrow 0 .
\end{aligned}
$$

Let $\operatorname{Ref}^{\prime}(R[x])=\operatorname{Ref}(R[x] /(\operatorname{Ref}(R)+\operatorname{Pro}(R[x]))$.

THEOREM 7. Let $R$ be a Noetherian integrally closed domain with quotient field $K$. Then the sequence

$$
0 \longrightarrow \operatorname{Ref}^{\prime}(R[x]) \longrightarrow B^{\prime}(R[x]) \longrightarrow B^{\prime}(K[x])
$$

is exact. If $K$ is perfect then $\operatorname{Ref}^{\prime}(R[x]) \cong B^{\prime}(R[x])$.

Proof. Let $\mathrm{Cl}(R)$ be the elements of $\operatorname{Ref}(R)$ of $\operatorname{rank}=1$ and let $\mathrm{Pic}(R)$ be the subgroup of projective modules in $\mathrm{Cl}(R)$. Consider the next diagram. By a theorem of $B$. Auslander (Theorem 2 in [8]) the first horizontal row is exact. By 7.19 p. 147 of [5] the first vertical row is exact. The last three vertical rows are split exact. Thus the bottom horizontal row is exact. If $K$ is perfect then $B^{\prime}(K[x])=0$ and $B^{\prime}(R[x]) \cong \operatorname{Ref}(R[x]) /(\operatorname{Ref}(R)+\operatorname{Pro}(R[x])) . \quad$ This completes the proof. 


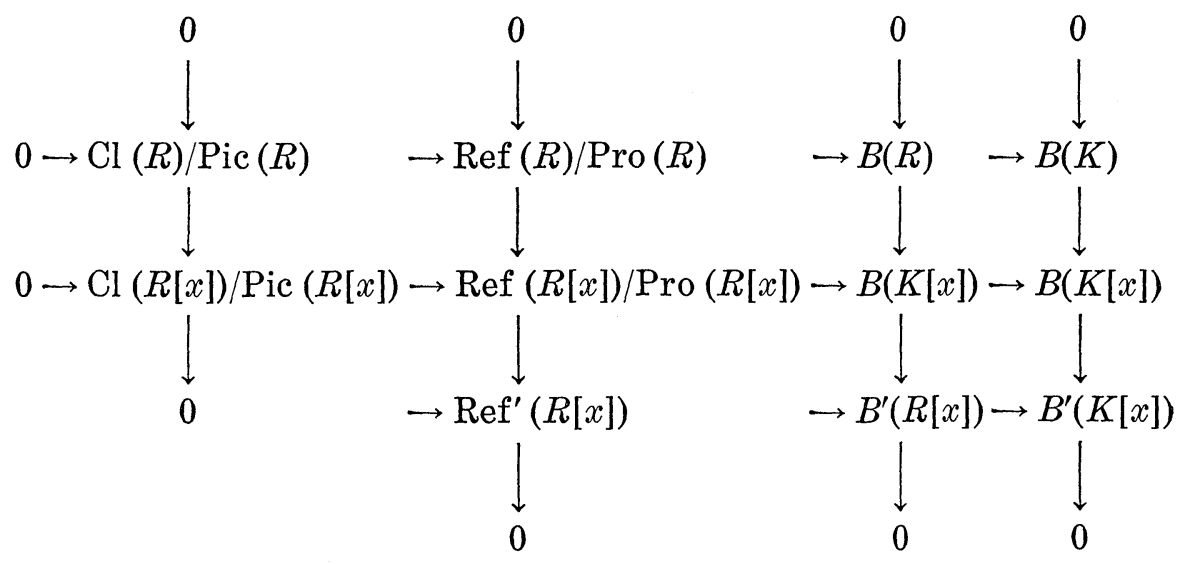

Let $R$ be a commutative von Neumann regular ring and let $\mathfrak{S}$ be the collection of ideals $I$ in $R$ maximal with respect to the property that $I$ is generated by idempotents. Then $\mathfrak{S}$ is the set of maximal ideals of $R$ and $R$ is isomorphic to a subring of $\prod_{I \in в} R / I$ (direct product).

THEOREM 8. Let $R$ be a commutative von Neumann regular ring, then $B(R) \cong B(R[x])$ if and only if $R / I$ is a perfect field for each maximal ideal $I$ of $R$. If $R$ is a Boolean ring then $B(R[x])=0$.

Proof. Consider the diagram

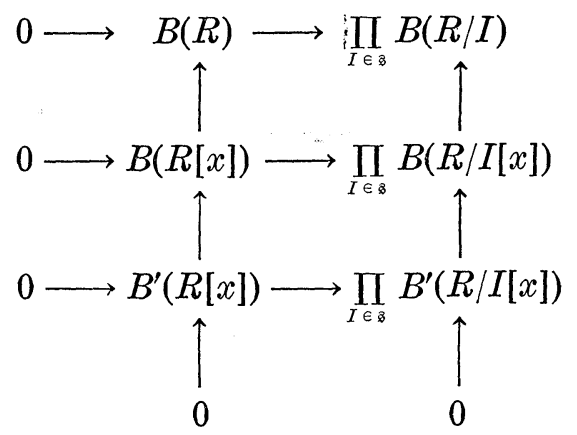

The idempotents of $R[x]$ are precisely the idempotents of $R$ so $\mathfrak{B}$ is the Boolean spectrum of $R$ and the set $\{I \cdot R[x] \mid I \in \mathfrak{B}\}$ is the Boolean spectrum of $R[x]$. In [15] it is shown that the first two rows of the diagram are exact, thus the third row is exact. If $R / I$ is perfect for all $I \in \mathfrak{B}$ then $\Pi_{I \in B} B^{\prime}(R / I[x])=0$ and $B^{\prime}(S[x])=0$.

If $B^{\prime}(R[x]) \neq 0$ then $B^{\prime}(R / I[x]) \neq 0$ for some $I$. For this $I$ the field $R / I$ is not perfect. If $R$ is a Boolean ring then $R$ is von Neumann regular and $R / I$ is the field with two elements for each $I \in \mathfrak{B}$. Thus 
$B(R) \cong B(R[x])$ and $B(R)=(0)$ so $B(R[x])=0$.

2. First we consider two examples also contained in [14]. Let $F$ be the algebraic closure of the field with $p$-elements and let $R=$ $F[t]$ be the ring of polynomials in one variable over $F$. Then $R$ is a principal ideal domain with a nonperfect quotient field $K$. By Theorem $7 B^{\prime}(R[x])$ is a subgroup of $B^{\prime}(K[x])$. On p. 390 of [3] a nontrivial element of $B^{\prime}(R[x])$ is given.

Next let $R=\left[Z \sqrt{2}\left(s^{2}+1\right), s\right]$ where $s$ is an indeterminate and $Z$ is the ring of rational integers. The integral closure $\bar{R}$ of $R$ is $Z[\sqrt{2,} s]$ and the conductor $c=\left(s^{2}+1\right) \bar{R}$. It is shown in [13] that $B^{\prime}(R[x]) \cong Z /(2)[t]$ but that $B(K / R) \cong B(\bar{R} / R)=0$ where $K$ is the quotient field of $R$. Now $K$ is a field of characteristic $=0$ so $B^{\prime}(K[x])=$ 0 and $B(K[x]) \rightarrow B(K(x))$ is a monomorphism.

Apply Lemma 5 with $S=K$ and we have

$$
B^{\prime}(R[x]) \cong B(K[x] / R[x]) \cong B(K[x] / R[x]) \cong Z /(2)[t]
$$

Thus $R$ is a domain with $B(R) \rightarrow B(K)$ a monomorphis yet $B(R[x]) \rightarrow$ $B(K[x])$ has an infinite kernal.

Next let $Z$ denote the rational integers, it is well known that $B(Z)=0$. Thus $B(Z[x])=0$ since $Z$ is a principal ideal domain of characteristic $=0$. However, $B(Z[x] /(2 x-1))=B(Z[1 / 2]) \neq 0$ since the ordinary quaternion algebra represents a nonzero class in $B(Z[1 / 2])$.

Now let $L$ be a finite extention of the perfect field $k$. Then $L \cong k[x] /(p(x))$ for some irreducible polynomial $p(x) \in k[x]$. Choose $k, L$ so that the natural map $B(k) \rightarrow B(L)$ is not onto. Then one has the commuting diagram

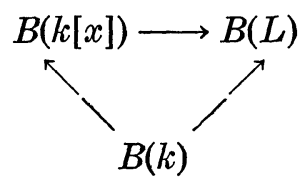

Since $B(k) \cong B(k[x])$ we have $B(k[x]) \rightarrow B(L)$ is not onto. The ideal generated by $p(x)$ is maximal in $k[x]$.

Next let $\bar{Z}_{p}$ be the $p$-adic completion of the ring $Z$ of integers for a finite prime $p$. Let $R$ be the completion of the localization at $(p)$ of $\bar{Z}_{p}|t|$. We get the following diagram

Also $R$ is a principal ideal domain of characteristic $=0$ so $B^{\prime}(R[x])=$ 0 . Since $B(R /(p)) \cong B(R)$ by Theorem 2 we have $B(R /(p) \cong B(R) \cong$ $B(R[x])$, and thus $K=0$. However $R /(p)$ is not a perfect field so $B^{\prime}(R /(p)[x]) \neq 0$ (p. 390 of [3]). Thus $B(R[x] \rightarrow B(R /(p)[x])$ is not onto. 


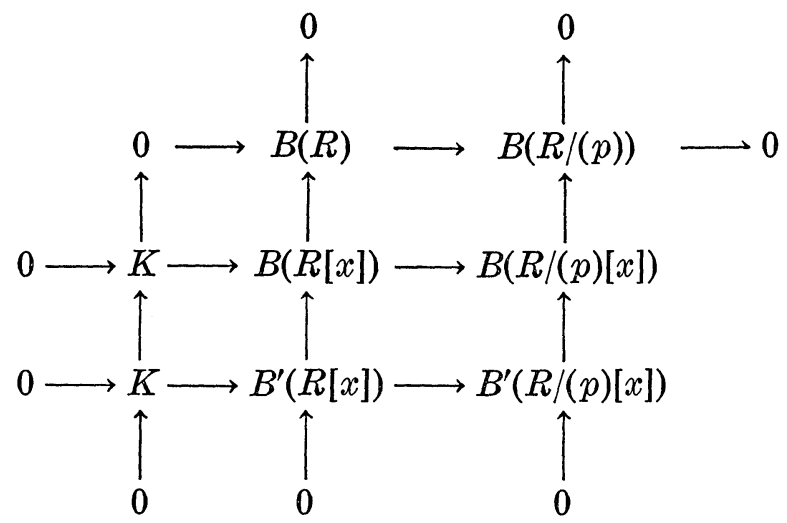

If $R$ is a local ring with maximal ideal $m$ is the induced homomorphism $B(R) \rightarrow B(R / m)$ onto? If $R$ is a Noetherian integrally closed domain with characteristic $R=0$ is $B(R) \cong B(R[x])$ ? If $R$ is a Noetherian integrally closed domain is $\operatorname{Ref}^{\prime}(R[x])=0$ ?

\section{REFERENCES}

1. B. Auslander, The Brauer group of a ringed space, J. of Algebra, 4 (1966), 220-273.

2. M. Auslander and O. Goldman, Maximal orders, Trans. Amer. Math. Soc. 97, (1960), 1-24.

3. - The Brauer group of a commutative ring, Trans. Amer. Math. Soc., 97 (1960), 367-409.

4. G. Azumaya On maximally central algebras, Nagoya Math. J., 2 (1951), 199-150.

5. H. Bass Algebraic K-Theory, W. A. Benjamin, New York (1968).

6. N. Bourbaki, Algebre Commutative, Chapitre 7, Hermann (1965).

7. L. Childs, Brauer groups of affine rings, Ring Theory-Proceedings of the Oklahoma Conference, Lecture Notes in Pure and Applied Mathematics 7, Marcel Dekker, Inc. N.Y., 1974, 83-92.

8. L. Childs, G. Garfinkel and M. Orzech, On the Brauer group and factoriality of normal domains, Queen's Mathematical Preprints 1974-9, Queens University, Kingston Ontario.

9. F. DeMeyer, The Brauer group of a ring modulo an ideal, Rocky Mountain J. Math., (to appear).

10. F. DeMeyer and E. Ingraham, Separable Algebras over Commutative Rings, Lecture Notes in Math \#181, Springer Verlag New York (1970).

11. S. Greco, Algebras over nonlocal Hensel rings II, J. of Algebra, 13 (1969), 48-56.

12. M. Greenberg, Lectures on Forms in Many Variables, Benjamin (1969).

13. M. A. Knus and M. Ojanguren, Théorie de la Descente et Algebres d'Azumaya, Lecture Notes in Mathematics \#389, Springer Verlag New York 1974).

14. M. Knus and M. Ojanguren, A Mayer-Victoirs sequence for the Brauer group, J. Pure and Applied Algebra. (to appear).

15. A. Magid, Pierce's representation and separable algebras, Illinois J. Math., 15 (1971), 114-121.

16. A. Roy and R. Sridharen, Derivations in Azumaya algebras, J. Math. Kyota U., 7 (1967), 161-167.

Received April 14, 1975.

Colorado State University 


\title{
PACIFIC JOURNAL OF MATHEMATICS
}

\section{EDITORS}

RichaRd ARENS (Managing Editor)

University of California

Los Angeles, California 90024

\section{R. A. Beaumont}

University of Washington

Seattle, Washington 98105
J. DUGUNDJI

Department of Mathematics University of Southern California Los Angeles, California 90007

D. Gilbarg and J. Milgram

Stanford University

Stanford, California 94305

\section{ASSOCIATE EDITORS}
E. F. BECKENBACH
B. H. NeUmanN
F. WOLF
K. YoshIDA

\section{SUPPORTING INSTITUTIONS}

\author{
UNIVERSITY OF BRITISH COLUMBIA \\ CALIFORNIA INSTITUTE OF TECHNOLOGY \\ UNIVERSITY OF CALIFORNIA \\ MONTANA STATE UNIVERSITY \\ UNIVERSITY OF NEVADA \\ NEW MEXICO STATE UNIVERSITY \\ OREGON STATE UNIVERSITY \\ UNIVERSITY OF OREGON \\ OSAKA UNIVERSITY
}

\author{
UNIVERSITY OF SOUTHERN CALIFORNIA \\ STANFORD UNIVERSITY \\ UNIVERSITY OF TOKYO \\ UNIVERSITY OF UTAH \\ WASHINGTON STATE UNIVERSITY \\ UNIVERSITY OF WASHINGTON \\ AMERICAN MATHEMATICAL SOCIETY
}

The Supporting Institutions listed above contribute to the cost of publication of this Journal, but they are not owners or publishers and have no responsibility for its content or policies.

Mathematical papers intended for publication in the Pacific Journal of Mathematics should be in typed form or offset-reproduced, (not dittoed), double spaced with large margins. Underline Greek letters in red, German in green, and script in blue. The first paragraph or two must be capable of being used separately as a synopsis of the entire paper. Items of the bibliography should not be cited there unless absolutely necessary, in which case they must be identified by author and Journal, rather than by item number. Manuscripts, in triplicate, may be sent to any one of the editors. Please classify according to the scheme of Math. Reviews, Index to Vol. 39. All other communications should be addressed to the managing editor, or Elaine Barth, University of California, Los Angeles, California, 90024.

The Pacific Journal of Mathematics expects the author's institution to pay page charges, and reserves the right to delay publication for nonpayment of charges in case of financial emergency.

100 reprints are provided free for each article, only if page charges have been substantially paid. Additional copies may be obtained at cost in multiples of 50 .

The Pacific Journal of Mathematics is issued monthly as of January 1966. Regular subscription rate: $\$ 72.00$ a year (6 Vols., 12 issues). Special rate: $\$ 36.00$ a year to individual members of supporting institutions.

Subscriptions, orders for back numbers, and changes of address should be sent to Pacific Journal of Mathematics, 103 Highland Boulevard, Berkeley, California, 94708.

PUBLISHED BY PACIFIC JOURNAL OF MATHEMATICS, A NON-PROFIT CORPORATION

Printed at Kokusai Bunken Insatsusha (International Academic Printing Co., Ltd.), 8-8, 3-chome, Takadanobaba, Shinjuku-ku, Tokyo 160, Japan.

Copyright (C) 1975 by Pacific Journal of Mathematics Manufactured and first issued in Japan 


\section{Pacific Journal of Mathematics}

\section{Vol. 59, No. $2 \quad$ June, 1975}

Aharon Atzmon, A moment problem for positive measures on the unit disc ........

Peter W. Bates and Grant Bernard Gustafson, Green's function inequalities for

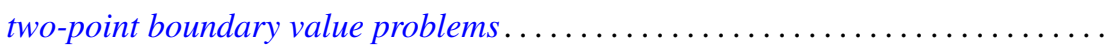

Howard Edwin Bell, Infinite subrings of infinite rings and near-rings ...........

Grahame Bennett, Victor Wayne Goodman and Charles Michael Newman, Norms of

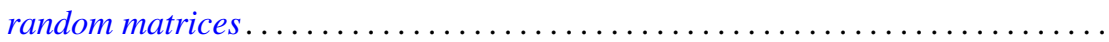

Beverly L. Brechner, Almost periodic homeomorphisms of $E^{2}$ are periodic.........

Beverly L. Brechner and R. Daniel Mauldin, Homeomorphisms of the plane ........

Jia-Arng Chao, Lusin area functions on local fields ......................

Frank Rimi DeMeyer, The Brauer group of polynomial rings ...............

M. V. Deshpande, Collectively compact sets and the ergodic theory of

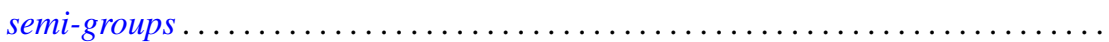

Raymond Frank Dickman and Jack Ray Porter, $\theta$-closed subsets of Hausdorff

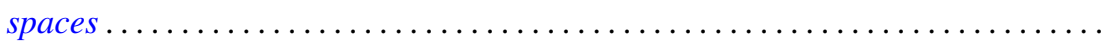

Charles P. Downey, Classification of singular integrals over a local field .......... 407

Daniel Reuven Farkas, Miscellany on Bieberbach group algebras . . . . . . . . . . . .

Peter A. Fowler, Infimum and domination principles in vector lattices . . . . . . . . . .

Barry J. Gardner, Some aspects of T-nilpotence. II: Lifting properties over

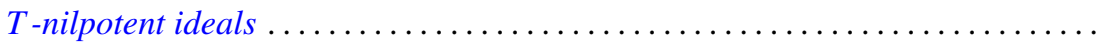

Gary Fred Gruenhage and Phillip Lee Zenor, Metrization of spaces with countable

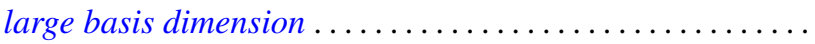

J. L. Hickman, Reducing series of ordinals...

Hugh M. Hilden, Generators for two groups related to the braid group ...

Tom (Roy Thomas Jr.) Jacob, Some matrix transformations on analytic sequence

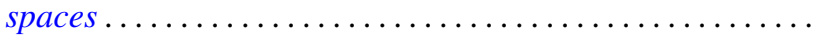

Elyahu Katz, Free products in the category of $k_{w}$-groups . .....

Tsang Hai Kuo, On conjugate Banach spaces with the Radon-Nikodým property...

Norman Eugene Liden, $K$-spaces, their antispaces and related mappings ...

Clinton M. Petty, Radon partitions in real linear spaces ........

Alan Saleski, A conditional entropy for the space of pseudo-Menger maps ....

Michael Singer, Elementary solutions of differential equations .

Eugene Spiegel and Allan Trojan, On semi-simple group algebras. I. . .

Charles Madison Stanton, Bounded analytic functions on a class of open Riemann

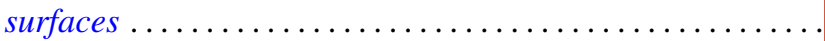

Sherman K. Stein, Transversals of Latin squares and their generalizations ....

Ivan Ernest Stux, Distribution of squarefree integers in non-linear sequences . . .

Lowell G. Sweet, On homogeneous algebras ................

Lowell G. Sweet, On doubly homogeneous algebras .......... .

Florian Vasilescu, The closed range modulus of operators ......

Arthur Anthony Yanushka, A characterization of the symplectic groups $\operatorname{PSp}(2 m, q)$

as rank 3 permutation groups... 